\title{
A NOTE ON TYPE 2 DEGENERATE MULTI-POLY-BERNOULLI POLYNOMIALS AND NUMBERS
}

\author{
WASEEM AHMAD KHAN, AYSHA KHAN, AND UGUR DURAN
}

\begin{abstract}
Inspired by the definition of degenerate multi-poly-Genocchi polynomials given by using the degenerate multi-polyexponential functions. In this paper, we consider a class of new generating function for the degenerate multi-poly-Bernoulli polynomials, called the type 2 degenerate multi-poly-Bernoulli polynomials by means of the degenerate multiple polyexponential functions. Then, we investigate their some properties and relations. We show that the type 2 degenerate multi-poly-Bernoulli polynomials equals a linear combination of the weighted degenerate Bernoulli polynomials and Stirling numbers of the first kind. Moreover, we provide an addition formula and a derivative formula. Furthermore, in a special case, we acquire a correlation between the type 2 degenerate multi-poly-Bernoulli numbers and degenerate Whitney numbers.
\end{abstract}

\section{INTRODUCTION}

Special polynomials has recently been applied in numerous fields of applied and pure mathematics besides in such other disciplines as physics, economics, statistics, probability theory, biology and engineering, $c f$. [1-24] and see also the references cited therein. Intense research activities in such an area as the theory of special polynomials are principally motivated by their importance in not only pure and applied mathematics but also mentioned other disciplines.

In 1956, Carlitz [1] considered a degenerate form of the well-known Staudt-Clausen theorem and then, in 1979, gave the degenerate versions of the Bernoulli, Stirling and Eulerian numbers in [2]. In spite of their being already more than sixth years old, these studies are still hot topic and today enveloped in an aura of mystery within the scientific community. From 1956 to the present, the degenerate versions of many polynomials, theorems, numbers etc. are investigated intensively by many mathematicians. In fact, in the most recent years, many researchers have been worked on degenerate type various numbers and polynomials. For example, the degenerate Hermite polynomials in [3], the degenerate Hermite-Bernoulli poynomials and numbers in [4], the degenerate Frobenius-Euler-Hermite polynomials in [5], the degenerate Hermite-polyBernoulli polynomials in [6], the degenerate Stirling polynmials of the second kind in [11], the degenerate Bernoulli polynomials of the second kind in [12] and the degenerate Bell polynomials in [13] and degenerate Genocchi polynomials in [19] have been considered and studied extensively.

In this paper, we introduce a novel class of degenerate multi-poly-Bernoulli polynomials and numbers by means of the degenerate multi-polyexponential function and studied their main explicit relations and identities. This work is organized as follows:

- Section 2 includes several known definitions and notations.

- In Section 3, we consider a novel class of degenerate multi-poly-Bernoulli polynomials and numbers and investigate their diverse properties and relations.

- The last section outlines finding gains and the conclusions in this work and mentions recommentations for future studies.

1991 Mathematics Subject Classification. Primary 11B73, Secondary 11B83, 05A19.

Key words and phrases. Bernoulli polynomials, Degenerate multi-polyexponential functions, Degenerate multi-polyBernoulli polynomials, Degenerate Stirling numbers, Degenerate Whitney numbers. 


\section{Preliminaries and Definitions}

Let $\mathbb{Z}$ denotes the set of all integers, $\mathbb{R}$ denotes the set of all real numbers and $\mathbb{C}$ denotes the set of all complex numbers. For any $\lambda \in \mathbb{R} /\{0\}$ (or $\mathbb{C} /\{0\}$ ), degenerate version of the exponential function $e_{\lambda}^{x}(t)$ is defined by the following series (see, $[1-6,11-21]$ )

$$
e_{\lambda}^{x}(t):=(1+\lambda t)^{\frac{1}{\lambda}}=\sum_{n=0}^{\infty}(x)_{n, \lambda} \frac{t^{n}}{n !},
$$

where $(x)_{0, \lambda}=1$ and $(x)_{n, \lambda}=x(x-\lambda) \cdots(x-(n-1) \lambda)$ for $n \geq 1$, cf. [1-6, 11-21].

It is obviously seen that $\lim _{\lambda \rightarrow 0} e_{\lambda}^{x}(t)=e^{x t}$. Note that $e_{\lambda}^{1}(t):=e_{\lambda}(t)$.

Carlitz [1] introduced the degenerate Bernoulli polynomials as follows

$$
\frac{t}{e_{\lambda}(t)-1} e_{\lambda}^{x}(t)=\sum_{n=0}^{\infty} \beta_{n}(x ; \lambda) \frac{t^{n}}{n !} .
$$

In the special case $x=0, \beta_{n}(0 ; \lambda):=\beta_{n}(\lambda)$ are called the degenerate Bernoulli numbers.

Note that

$$
\lim _{\lambda \longrightarrow 0} \beta_{n}(x ; \lambda)=B_{n}(x),
$$

where $B_{n}(x)$ are the familar Bernoulli polynomials given by the following generating function

$$
\frac{t}{e^{t}-1} e^{x t}=\sum_{n=0}^{\infty} B_{n}(x) \frac{t^{n}}{n !}, \quad(|t|<2 \pi) .
$$

The polyexponential functions are defined by (see [10])

$$
\operatorname{Ei}_{k}(x)=\sum_{n=1}^{\infty} \frac{x^{n}}{(n-1) ! n^{k}}, \quad(k \in \mathbb{Z}) .
$$

For $k=1$ in 2.3 , we have $\operatorname{Ei}_{1}(x)=e^{x}-1$.

The modified degenerate polyexponential functions [14] are defined by

$$
\operatorname{Ei}_{k, \lambda}(x)=\sum_{n=1}^{\infty} \frac{(1)_{n, \lambda}}{(n-1) ! n^{k}} x^{n}
$$

It is note that for $k=1, \operatorname{Ei}_{1, \lambda}(x)=e_{\lambda}(x)-1$.

Let $k \in \mathbb{Z}$. The type 2 degenerate poly-Bernoulli polynomials [14] are defined by means of the following generating function

$$
\frac{\operatorname{Ei}_{k, \lambda}\left(\log _{\lambda}(1+t)\right)}{e_{\lambda}(t)-1} e_{\lambda}^{x}(t)=\sum_{n=0}^{\infty} B_{n, \lambda}^{(k)}(x) \frac{t^{n}}{n !},
$$

where $\log _{\lambda}(1+t)$ is the degenerate version of the logarithm function given by (cf. [14])

$$
\log _{\lambda}(1+t)=\sum_{n=1}^{\infty} \lambda^{n-1}(1)_{n, 1 / \lambda} \frac{t^{n}}{n !}, \quad(\lambda \in \mathbb{R})
$$

which is also the inverse function of the degenerate exponential function $e_{\lambda}(t)$ as shown below

$$
e_{\lambda}\left(\log _{\lambda}(1+t)\right)=\log _{\lambda}\left(e_{\lambda}(1+t)\right)=1+t .
$$

When $x=0, B_{n, \lambda}^{(k)}=B_{n, \lambda}^{(k)}(0)$ are called the type 2 degenerate poly-Bernoulli numbers.

For $k_{1}, k_{2}, \cdots k_{r} \in \mathbb{Z}$, the multiple polylogarithm function [23] is defined by

$$
\operatorname{Ei}_{k_{1}, k_{2}, \cdots, k_{r}}(x)=\sum_{0<n_{1}<n_{2}<\cdots<n_{r}} \frac{x^{n_{r}}}{n_{1}^{k_{1}} n_{2}^{k_{2}} \cdots n_{r}^{k_{r}}}
$$

where the sum is over all integers $n_{1}, n_{2}, \cdots, n_{r}$ satisfying $0<n_{1}<n_{2}<\cdots<n_{r}$. 
By means of the multiple polylogarithm function, the multi-poly-Bernoulli polynomials are given by

$$
\frac{r ! \operatorname{Ei}_{k_{1}, k_{2}, \cdots, k_{r}}(\log (1+t))}{\left(e^{t}-1\right)^{r}} e^{x t}=\sum_{n=0}^{\infty} B_{n}^{\left(k_{1}, k_{2}, \cdots, k_{r}\right)}(x) \frac{t^{n}}{n !} .
$$

Also, the degenerate version of the multi-poly-Bernoulli polynomials are defined as given below

$$
\frac{r ! \operatorname{Ei}_{k_{1}, k_{2}, \cdots, k_{r}}\left(1-e^{-t}\right)}{\left(e_{\lambda}(t)-1\right)^{r}} e_{\lambda}^{x}(t)=\sum_{n=0}^{\infty} \beta_{n, \lambda}^{\left(k_{1}, k_{2}, \cdots k_{r}\right)}(x) \frac{t^{n}}{n !}, \text { see [20]). }
$$

The degenerate Stirling numbers of the first kind $(c f .[14,19])$ and second kind $(c f .[4,5,6,18-22])$ are defined, respectively, by

$$
\frac{1}{k !}\left(\log _{\lambda}(1+t)\right)^{k}=\sum_{n=k}^{\infty} S_{1, \lambda}(n, k) \frac{t^{n}}{n !}, \quad(k \geq 0)
$$

and

$$
\frac{1}{k !}\left(e_{\lambda}(t)-1\right)^{k}=\sum_{n=k}^{\infty} S_{2, \lambda}(n, k) \frac{t^{n}}{n !},(k \geq 0) .
$$

Noting here that as $\lambda \rightarrow 0$, the degenerate Stirling numbers of the first kind and second kind reduce to the usual Stirling numbers of the first kind $S_{1}(n, k)$ and second kind $S_{2}(n, k)$ as follows

$$
\frac{1}{k !}(\log (1+t))^{k}=\sum_{n=k}^{\infty} S_{1}(n, k) \frac{t^{n}}{n !},(k \geq 0), \quad(c f .[14,19])
$$

and

$$
\frac{1}{k !}\left(e^{t}-1\right)^{k}=\sum_{n=k}^{\infty} S_{2}(n, k) \frac{t^{n}}{n !},(k \geq 0), \quad(c f .[4,5,6,18-22]) .
$$

\section{Type 2 Degenerate Multi-Poly-Bernoulli Polynomials}

Let $k_{1}, k_{2}, \cdots, k_{r} \in \mathbb{Z}$. The degenerate multiple polyexponential function is defined by

$$
\operatorname{Ei}_{k_{1}, k_{2}, \cdots, k_{r}, \lambda}(x)=\sum_{0<n_{1}<n_{2}<\cdots<n_{r}} \frac{(1)_{n_{1}, \lambda} \cdots(1)_{n_{r}, \lambda} x^{n_{r}}}{\left(n_{1}-1\right) ! \cdots\left(n_{r}-1\right) ! n_{1}^{k_{1}} \cdots n_{r}^{k_{r}}}, \text { (see [21]), }
$$

where the sum is over all integers $n_{1}, n_{2}, \cdots, n_{r}$ satisfying $0<n_{1}<n_{2}<\cdots<n_{r}$. By means of this function, Kim et al. [21] introduced and studied the degenerate multi-poly-Genocchi polynomials given by

$$
\frac{r ! \operatorname{Ei}_{k_{1}, k_{2}, \cdots, k_{r}, \lambda}\left(\log _{\lambda}(1+t)\right)}{\left(e_{\lambda}(t)+1\right)^{r}} e_{\lambda}^{x}(t)=\sum_{n=0}^{\infty} g_{n, \lambda}^{\left(k_{1}, k_{2}, \cdots k_{r}\right)}(x) \frac{t^{n}}{n !} .
$$

Inspired by the definition of degenerate multi-poly-Genocchi polynomials, using the degenerate multiple polyexponential function (3.1), we give the following definition.

Definition 1. Let $k \in \mathbb{Z}$. Type 2 degenerate multi-poly-Bernoulli polynomials are defined by the following exponential generating function:

$$
\frac{r ! \mathrm{Ei}_{k_{1}, k_{2}, \cdots, k_{r}, \lambda}\left(\log _{\lambda}(1+t)\right)}{\left(e_{\lambda}(t)-1\right)^{r}} e_{\lambda}^{x}(t)=\sum_{n=0}^{\infty} \beta_{n, \lambda}^{\left(k_{1}, k_{2}, \cdots, k_{r}\right)}(x) \frac{t^{n}}{n !} .
$$

In the case $x=0$ in (3.3), the type 2 degenerate multi-poly-Bernoulli polynomials reduce to the corresponding numbers, namely the type 2 degenerate multi-poly-Bernoulli numbers $\beta_{n, \lambda}^{\left(k_{1}, k_{2}, \cdots, k_{r}\right)}(0):=\beta_{n, \lambda}^{\left(k_{1}, k_{2}, \cdots, k_{r}\right)}$. 
Remark 1. As $\lambda \rightarrow 0$, the type 2 degenerate multi-poly-Bernoulli polynomials reduce to the multi-polyBernoulli polynomials (2.7).

$$
\lim _{\lambda \rightarrow 0} \beta_{n, \lambda}^{\left(k_{1}, k_{2}, \cdots, k_{r}\right)}(x)=B_{n}^{\left(k_{1}, k_{2}, \cdots, k_{r}\right)}(x), \quad(n \geq 0) .
$$

From Definition 1, we observe that

$$
\begin{aligned}
\sum_{n=0}^{\infty} \beta_{n, \lambda}^{\left(k_{1}, k_{2}, \cdots k_{r}\right)}(x) \frac{t^{n}}{n !} & =\frac{r ! \operatorname{Ei}_{k_{1}, k_{2}, \cdots, k_{r}, \lambda}\left(\log _{\lambda}(1+t)\right)}{\left(e_{\lambda}(t)-1\right)^{r}} e_{\lambda}^{x}(t) \\
& =\sum_{n=0}^{\infty} \beta_{n, \lambda}^{\left(k_{1}, k_{2}, \cdots k_{r}\right)} \frac{t^{n}}{n !} \sum_{m=0}^{\infty}(x)_{m, \lambda} \frac{t^{m}}{m !} \\
& =\sum_{n=0}^{\infty}\left(\sum_{m=0}^{n}\left(\begin{array}{c}
n \\
m
\end{array}\right) \beta_{n-m, \lambda}^{\left(k_{1}, k_{2}, \cdots k_{r}\right)}(x)_{m, \lambda}\right) \frac{t^{n}}{n !}
\end{aligned}
$$

which gives the following theorem.

Theorem 1. The following relationship

$$
\beta_{n, \lambda}^{\left(k_{1}, k_{2}, \cdots k_{r}\right)}(x)=\sum_{m=0}^{n}\left(\begin{array}{c}
n \\
m
\end{array}\right) \beta_{n-m, \lambda}^{\left(k_{1}, k_{2}, \cdots k_{r}\right)}(x)_{m, \lambda}
$$

hold true for $n \geq 0$.

The weighted degenerate Bernoulli polynomials are given by the following series expansion:

$$
\sum_{n=0}^{\infty} \beta_{n}^{(r)}(x ; \lambda) \frac{t^{n}}{n !}=\left(\frac{t}{e_{\lambda}(t)-1}\right)^{r} e_{\lambda}^{x}(t),
$$

cf. $[3,4,6,12,14]$.

From Definition 1 and (2.9), we see that

$$
\begin{aligned}
& \sum_{n=0}^{\infty} \beta_{n, \lambda}^{\left(k_{1}, k_{2}, \cdots, k_{r}\right)}(x) \frac{t^{n}}{n !}=\frac{r ! e_{\lambda}^{x}(t)}{\left(e_{\lambda}(t)-1\right)^{r}} \sum_{0<n_{1}<n_{2}<\cdots<n_{r}} \frac{(1)_{n_{1}, \lambda} \cdots(1)_{n_{r}, \lambda}\left(\log _{\lambda}(1+t)\right)^{n_{r}}}{\left(n_{1}-1\right) ! \cdots\left(n_{r}-1\right) ! n_{1}^{k_{1}} \cdots n_{r}^{k_{r}}} \\
& =\frac{r ! e_{\lambda}^{x}(t)}{\left(e_{\lambda}(t)-1\right)^{r}} \sum_{0<n_{1}<n_{2}<\cdots<n_{r}} \frac{(1)_{n_{1}, \lambda} \cdots(1)_{n_{r}, \lambda} n_{r} !}{\left(n_{1}-1\right) ! \cdots\left(n_{r}-1\right) ! n_{1}^{k_{1}} \cdots n_{r-1}^{k_{r-1}} n_{r}^{k_{r}}} \sum_{m=n_{r}}^{\infty} S_{1 . \lambda}\left(m, n_{r}\right) \frac{t^{m}}{m !} \\
& =\frac{r !}{t^{r}}\left(\frac{t^{r} e_{\lambda}^{x}(t)}{\left(e_{\lambda}(t)-1\right)^{r}}\right) \sum_{m=n_{r}}^{\infty}\left(\sum_{0<n_{1}<n_{2}<\cdots<n_{r}} \frac{(1)_{n_{1}, \lambda} \cdots(1)_{n_{r}, \lambda} S_{1 . \lambda}\left(m, n_{r}\right) n_{r} !}{\left(n_{1}-1\right) ! \cdots\left(n_{r}-1\right) ! n_{1}^{k_{1}} \cdots n_{r-1}^{k_{r}-1} n_{r}^{k_{r}}}\right) \frac{t^{m}}{m !} \\
& =\frac{r !}{t^{r}} \sum_{l=0}^{\infty} \beta_{l}^{(r)}(x ; \lambda) \frac{t^{l}}{l !} \sum_{m=n_{r}}^{\infty}\left(\sum_{0<n_{1}<n_{2}<\cdots<n_{r}} \frac{(1)_{n_{1}, \lambda} \cdots(1)_{n_{r}, \lambda} S_{1 . \lambda}\left(m, n_{r}\right) n_{r} !}{\left(n_{1}-1\right) ! \cdots\left(n_{r}-1\right) ! n_{1}^{k_{1}} \cdots n_{r-1}^{k_{r-1}} n_{r}^{k_{r}}}\right) \frac{t^{m}}{m !} \\
& =\frac{1}{t^{r}} \sum_{n=0}^{\infty} \sum_{m=0}^{n} \sum_{0<n_{1}<n_{2}<\cdots<n_{r}} \frac{\left(\begin{array}{c}
n \\
m
\end{array}\right) r ! n_{r} !(1)_{n_{1}, \lambda} \cdots(1)_{n_{r}, \lambda}}{\left(n_{1}-1\right) ! \cdots\left(n_{r}-1\right) ! n_{1}^{k_{1}} \cdots n_{r-1}^{k_{r-1}} n_{r}^{k_{r}}} \beta_{n-m}^{(r)}(x ; \lambda) S_{1 . \lambda}\left(n-m, n_{r}\right) \frac{t^{n}}{n !},
\end{aligned}
$$

which means the following theorem.

Therefore, by equation (2.4), we obtain the following theorem. 
Theorem 2. For $k_{1}, k_{2}, \cdots k_{r} \in \mathbb{Z}$, and $n, r \in \mathbb{N}$ with $n \geq r$, we have

$$
\begin{aligned}
\beta_{n, \lambda}^{\left(k_{1}, k_{2}, \cdots, k_{r}\right)}(x)= & \sum_{m=0}^{n-r} \sum_{0<n_{1}<n_{2}<\cdots<n_{r}} \frac{n ! r ! n_{r} !(1)_{n_{1}, \lambda} \cdots(1)_{n_{r}, \lambda}}{m !(n-m-r) !} \\
& \times \frac{\beta_{n-r-m}^{(r)}(x ; \lambda) S_{1 . \lambda}\left(n-r-m, n_{r}\right)}{\left(n_{1}-1\right) ! \cdots\left(n_{r}-1\right) ! n_{1}^{k_{1}} \cdots n_{r-1}^{k_{r}-1} n_{r}^{k_{r}}}
\end{aligned}
$$

In view of Definition 1 , we see that

$$
\begin{gathered}
\sum_{n=0}^{\infty} \beta_{n, \lambda}^{\left(k_{1}, k_{2}, \cdots, k_{r}\right)}(x+y) \frac{t^{n}}{n !}=\frac{r ! \operatorname{Ei}_{k_{1}, k_{2}, \cdots, k_{r}, \lambda}\left(\log _{\lambda}(1+t)\right)}{\left(e_{\lambda}(t)-1\right)^{r}} e_{\lambda}^{x+y}(t) \\
=\sum_{n=0}^{\infty} \beta_{n, \lambda}^{\left(k_{1}, k_{2}, \cdots, k_{r}\right)}(x) \frac{t^{n}}{n !} \sum_{m=0}^{\infty}(y)_{m, \lambda} \frac{t^{m}}{m !} \\
=\sum_{n=0}^{\infty}\left(\sum_{m=0}^{n}\left(\begin{array}{c}
n \\
m
\end{array}\right)(y)_{m, \lambda} \beta_{n-m, \lambda}^{\left(k_{1}, k_{2}, \cdots, k_{r}\right)}(x)\right) \frac{t^{n}}{n !}
\end{gathered}
$$

which implies the following theorem includes the addtion formula for the type 2 degenerate multi-polyBernoulli polynomials.

Theorem 3. The following addition formula

$$
\beta_{n, \lambda}^{\left(k_{1}, k_{2}, \cdots, k_{r}\right)}(x+y)=\sum_{m=0}^{n}\left(\begin{array}{c}
n \\
m
\end{array}\right)(y)_{m, \lambda} \beta_{n-m, \lambda}^{\left(k_{1}, k_{2}, \cdots, k_{r}\right)}(x)
$$

is valid for $k_{1}, k_{2}, \cdots, k_{r} \in \mathbb{Z}$ and $n \geq 0$.

To invetigate the derivative property of the type 2 degenerate multi-poly-Bernoulli polynomials, we now consider that

$$
\begin{aligned}
\sum_{n=0}^{\infty} \frac{d}{d x} \beta_{n, \lambda}^{\left(k_{1}, k_{2}, \cdots, k_{r}\right)}(x) \frac{t^{n}}{n !} & =\frac{r ! \operatorname{Ei}_{k_{1}, k_{2}, \cdots, k_{r}, \lambda}\left(\log _{\lambda}(1+t)\right)}{\left(e_{\lambda}(t)-1\right)^{r}} \frac{d}{d x} e_{\lambda}^{x}(t) \\
& =\sum_{n=0}^{\infty} \beta_{n, \lambda}^{\left(k_{1}, k_{2}, \cdots, k_{r}\right)}(x) \frac{t^{n}}{n !} \frac{1}{\lambda} \ln (1+\lambda t) \\
& =\left(\sum_{n=0}^{\infty} \beta_{n, \lambda}^{\left(k_{1}, k_{2}, \cdots, k_{r}\right)}(x) \frac{t^{n}}{n !}\right) \sum_{l=1}^{\infty} \frac{(-1)^{l+1}}{l} \lambda^{l-1} t^{l} \\
& =\sum_{n=0}^{\infty} \sum_{l=1}^{\infty} \beta_{n, \lambda}^{\left(k_{1}, k_{2}, \cdots, k_{r}\right)}(x) \frac{(-1)^{l+1}}{l} \lambda^{l-1} \frac{t^{n+l}}{n !},
\end{aligned}
$$

which provides the following theorem.

Theorem 4. The following relation

$$
\frac{d}{d x} \beta_{n, \lambda}^{\left(k_{1}, k_{2}, \cdots, k_{r}\right)}(x)=n ! \sum_{l=1}^{\infty} \beta_{n-l, \lambda}^{\left(k_{1}, k_{2}, \cdots, k_{r}\right)}(x) \frac{(-1)^{l+1}}{(n-l) ! l} \lambda^{l-1}
$$

is valid for $k_{1}, k_{2}, \cdots, k_{r} \in \mathbb{Z}$ and $n \geq 0$. 
By means of Definition 1, we attain that

$$
\begin{gathered}
\sum_{n=0}^{\infty} \beta_{n, \lambda}^{\left(k_{1}, k_{2}, \cdots, k_{r}\right)}(x) \frac{t^{n}}{n !}=\frac{r ! \operatorname{Ei}_{k_{1}, k_{2}, \cdots, k_{r}, \lambda}\left(\log _{\lambda}(1+t)\right)}{\left(e_{\lambda}(t)-1\right)^{r}} e_{\lambda}^{x}(t) \\
=\frac{r ! \operatorname{Ei}_{k_{1}, k_{2}, \cdots, k_{r}, \lambda}\left(\log _{\lambda}(1+t)\right)}{\left(e_{\lambda}(t)-1\right)^{r}}\left(e_{\lambda}(t)-1+1\right)^{x} \\
=\frac{r ! \operatorname{Ei}_{k_{1}, k_{2}, \cdots, k_{r}, \lambda}\left(\log _{\lambda}(1+t)\right)}{\left(e_{\lambda}(t)-1\right)^{r}} \sum_{l=0}^{\infty}\left(\begin{array}{c}
x \\
l
\end{array}\right)\left(e_{\lambda}(t)-1\right)^{l} \\
=\sum_{n=0}^{\infty} \beta_{n, \lambda}^{\left(k_{1}, k_{2}, \cdots, k_{r}\right)} \frac{t^{n}}{n !} \sum_{l=0}^{\infty}(x)_{l} \sum_{m=l}^{\infty} S_{2, \lambda}(m, l) \frac{t^{m}}{m !} \\
=\sum_{n=0}^{\infty}\left(\sum_{m=0}^{n} \sum_{l=0}^{m}\left(\begin{array}{c}
n \\
m
\end{array}\right)(x)_{l} S_{2, \lambda}(m, l) \beta_{n-m, \lambda}^{\left(k_{1}, k_{2}, \cdots, k_{r}\right)}\right) \frac{t^{n}}{n !}
\end{gathered}
$$

where the notation $(x)_{l}$ is falling factorial and is defined by $(x)_{0}=1$ and $(x)_{n}=x(x-1) \cdots(x-(n-1))$ for $n \geq 1$, cf. [1-6, 11-21]. Therefore, we arrive at the following theorem.

Theorem 5. The following correlation

$$
\beta_{n, \lambda}^{\left(k_{1}, k_{2}, \cdots, k_{r}\right)}(x)=\sum_{m=0}^{n} \sum_{l=0}^{m}\left(\begin{array}{c}
n \\
m
\end{array}\right)(x)_{l} S_{2, \lambda}(m, l) \beta_{n-m, \lambda}^{\left(k_{1}, k_{2}, \cdots, k_{r}\right)} .
$$

is valid for $k_{1}, k_{2}, \cdots, k_{r} \in \mathbb{Z}$ and $n \geq 0$.

Kim [11] introduced the degenerate Whitney numbers which are defined by the generating function to be

$$
\frac{\left(e_{\lambda}^{m}(t)-1\right)^{k}}{m^{k} k !} e_{\lambda}^{\alpha}(t)=\sum_{n=k}^{\infty} W_{m, \alpha}(n, k \mid \lambda) \frac{t^{n}}{n !},(k \geq 0) .
$$

Using Definition 1, we acquire that

$$
\begin{gathered}
\sum_{n=0}^{\infty} \beta_{n, \lambda}^{\left(k_{1}, k_{2}, \cdots, k_{r}\right)}(x u+\alpha) \frac{t^{n}}{n !}=\frac{r ! \operatorname{Ei}_{k_{1}, k_{2}, \cdots, k_{r}, \lambda}\left(\log _{\lambda}(1+t)\right)}{\left(e_{\lambda}(t)-1\right)^{r}} e_{\lambda}^{\alpha}(t) e_{\lambda}^{x u}(t) \\
=\frac{r ! \operatorname{Ei}_{k_{1}, k_{2}, \cdots, k_{r}, \lambda}\left(\log _{\lambda}(1+t)\right)}{\left(e_{\lambda}(t)-1\right)^{r}} e_{\lambda}^{\alpha}(t)\left(e_{\lambda}^{u}(t)-1+1\right)^{x} \\
=\frac{r ! \operatorname{Ei}_{k_{1}, k_{2}, \cdots, k_{r}, \lambda}\left(\log _{\lambda}(1+t)\right)}{\left(e_{\lambda}(t)-1\right)^{r}} e_{\lambda}^{\alpha}(t) \sum_{l=0}^{\infty}\left(\begin{array}{l}
x \\
l
\end{array}\right)\left(e_{\lambda}^{u}(t)-1\right)^{l} \\
=\frac{r ! \operatorname{Ei}_{k_{1}, k_{2}, \cdots, k_{r}, \lambda}\left(\log _{\lambda}(1+t)\right)}{\left(e_{\lambda}(t)-1\right)^{r}} \sum_{l=0}^{\infty} u^{l}(x)_{l} \frac{\left(e_{\lambda}^{u}(t)-1\right)^{l}}{l ! u^{l}} e_{\lambda}^{\alpha}(t) \\
=\frac{r ! \operatorname{Ei}_{k_{1}, k_{2}, \cdots, k_{r}, \lambda}\left(\log _{\lambda}(1+t)\right)}{\left(e_{\lambda}(t)-1\right)^{r}} \sum_{l=0}^{\infty} u^{l}(x)_{l} \frac{\left(e_{\lambda}^{u}(t)-1\right)^{l}}{l ! u^{l}} e_{\lambda}^{\alpha}(t) \\
=\sum_{n=0}^{\infty} \beta_{n, \lambda}^{\left(k_{1}, k_{2}, \cdots, k_{r}\right)} \frac{t^{n}}{n !} \sum_{n=0}^{\infty} \sum_{l=0}^{n} u^{l}(x)_{l} W_{u, \alpha}(n, l \mid \lambda) \frac{t^{n}}{n !} \\
=\sum_{n=0}^{\infty}\left(\sum_{m=0}^{n} \sum_{l=0}^{m}\left(\begin{array}{c}
n \\
m
\end{array}\right) u^{l}(x)_{l} W_{u, \alpha}(m, l \mid \lambda) \beta_{n-m, \lambda}^{\left(k_{1}, k_{2}, \cdots, k_{r}\right)}\right) \frac{t^{n}}{n !}
\end{gathered}
$$

which gives the last theorem as follows. 
Theorem 6. For $k_{1}, k_{2}, \cdots k_{r} \in \mathbb{Z}$ and $n \geq 0$, we have

$$
\beta_{n, \lambda}^{\left(k_{1}, k_{2}, \cdots, k_{r}\right)}(x \beta+\alpha)=\sum_{m=0}^{n} \sum_{l=0}^{m}\left(\begin{array}{c}
n \\
m
\end{array}\right) u^{l}(x)_{l} W_{u, \alpha}(m, l \mid \lambda) \beta_{n-m, \lambda}^{\left(k_{1}, k_{2}, \cdots, k_{r}\right)} .
$$

\section{Conclusions}

Motivated and inspired by the definition of the degenerate multi-poly-Genocchi polynomials introduced by Kim et al. [21], in the present paper, we have considered a class of new generating function for the degenerate multi-poly-Bernoulli polynomials, called the type 2 degenerate multi-poly-Bernoulli polynomials, by means of the degenerate multi-polylogarithm function. Then, we have derived some useful relations and properties. We have shown that the type 2 degenerate multi-poly-Bernoulli polynomials equals a linear combination of the degenerate poly-Bernoulli polynomials and Stirling numbers of the first and second kind. In a special case, we have investigated a correlation between the type 2 degenerate multi-poly-Bernoulli numbers and degenerate Whitney numbers.

In the future plans, we will continue to study degenerate versions of certain special polynomials and numbers and their applications to probability, physics, and engineering in addition to mathematics.

Author Contributions: All authors contributed equally to the manuscript and typed, read, and approved final manuscript.

Conflict of Interest: The authors declare no conflict of interest.

\section{REFERENCES}

[1] Carlitz, L. Degenerate Stirling, Bernoulli and Eulerian numbers. Utilitas Math. 1979, 15, 51-88.

[2] Carlitz, L. A degenerate Staudt-Clausen theorem, Arch. Math. (Basel) 1956, 7, 28-33.

[3] Haroon, H.; Khan, W.A. Degenerate Bernoulli numbers and polynomials associated with degenerate Hermite polynomials. Commun. Korean. Math. Soc. 2018, 33(2), 651-669.

[4] Khan, W.A. Degenerate Hermite-Bernoulli numbers and polynomials of the second kind, Prespacetime Journal. 2016, 7(9), 1297-1305.

[5] Khan, W.A. A new class of degenerate Frobenius-Euler-Hermite polynomials. Adv. Stud. Contemp. Math. (Kyungshang). 2018, 28(4), 567-576.

[6] Khan, W.A. A note on degenerate Hermite-poly-Bernoulli numbers and polynomials. J. Classical Anal. 2016, 8(1), 65-76.

[7] Khan, W.A.; Ghayasuddin, M.; Shadab, M. Multiple poly-Bernoulli polynomials of the second kind associated with Hermite polynomials. Fasciculi Mathematici. 2017, 58, 97-112.

[8] Khan, W.A.; Ghayasuddin, M.; Khan, N.; Choi, J. The 3-variable Hermite poly-Bernoulli polynomials of the second kind. Nonlinear Func. Anal. Appl. 2017, 22(5), 1013-1027.

[9] Kim, D.S.; Kim, T. Some identities for Bernoulli numbers of the second kind arising from a non-linear differential equation. Bull. Korean Math. Soc. 2015, 52(6), 2001-2010.

[10] Kim, D.S.; Kim, T. A note on polyexponential and unipoly functions. Russ. J. Math. Phys. bf 2019, 26(1), 40-49.

[11] Kim, T. A note on degenerate Stirling polynomials of the second kind. Proc. Jangjeon Math. Soc. 2017, 20(3), 319-331.

[12] Kim, T.; Jang,L.-C.; Kim, D.S.; Kim, H.-Y. Some identities on type 2 degenerate Bernoulli polynomials of the second kind. Symmetry. 2020, 12(4), Article ID 510(2020).

[13] Kim, T.; Kim, D.S. Degenerate polyexponential functions and degenerate Bell polynomials. J. Math. Anal. Appl. 2020, 487(2), 124017. 
[14] Kim, D.S.; Kim, T. A note on a new type of degenerate Bernoulli numbers. Russ. J. Math. Phys. 2020, 27(2), 227-235.

[15] Kim, T.; Kim, D.S.; Kim, H.Y.; Kwon, J. Degenerate Stirling polynomials of the second kind and some applications. Symmetry. 2019, 11(8), Article ID 1046.

[16] Kim, T.; Kim, D.S. Degenerate Laplace transform and degenerate gamma function. Russ. J. Math. Phys. 2017, 24(2), 241-248.

[17] Kim, T.; Khan, W.A.; Sharma, S.K.; Ghayasuddin, M. A note on parametric kinds of the degenerate poly-Bernoulli and poly-Genocchi polynomials. Symmetry. 2020, 12(4), Article ID 614.

[18] Kim, T.; Kim, D.S.; Kwon, J.; Lee, H. Degenerate polyexpnential functions and type 2 degenerate poly-Bernoulli numbers and polynomials. Adv. Differ. Equ. 2020, 2020:168, 12pp.

[19] Kim, T.; Kim, D.S.; Kwon, J.; Kim, H.Y. A note on degenerate Genocchi and poly-Genocchi numbers and polynomials. J. Ineq. Appl. 2020, 2020:110, 13pp.

[20] Kim, T.; Kim, D.S.; A note on degenerate multi-poly-Bernoulli numbers and polynomials. arXiv:2005.07319v1[math. NT] 15 May 2020.

[21] Kim, T.; Kim, D.S.; Kim, H.-Y.; Kwon, J. A note on degenerate multi-poly-Genocchi polynomials. Adv. Stud. Contemp. Math. (Kyungshang). 2020, 30(3), 447-454.

[22] Kim, T.; Kwon, H.-I.; Lee, S.-H; Seo, J.I. A note on poly-Bernoulli numbers and polynomials of the second kind. Adv. Diiffe. Eqn. 2014, 2014:219.

[23] Qi, F.; Kim, D.S.; Kim, T. Multiple poly-Bernoulli polynomials of the second kind. Adv. Stud. Contemp. Math. (Kyungshang). 2015, 25, 1-7.

[24] Roman, S. The umbral calculus. Pure App. Math. Vol. 111. Academic Press, Inc., New York, 1984.

Department of Mathematics and Natural Sciences, Prince Mohammad Bin fahd University, P.O Box 1664, Al Khobar 31952, Saudi Arabia

E-mail address: wkhan1@pmu.edu.sa

Department of Mathematics, College of Arts and Science-Wadi Al dawasir, Prince Sattam Bin Abdulaziz UniVersity, Riyadh Region 11991 Saudi Arabia

E-mail address: a.aysha@psau.edu.sa

Department of the Basic Concepts of Engineering, Faculty of Engineering and Natural Sciences, Iskenderun TeChnical University, TR-31200 Hatay, Turkey

E-mail address: mtdrnugur@gmail.com \& ugur.duran@iste.edu.tr 